\title{
Desafios da pandemia para a mentoria: o papel dos mentores juniores e das redes sociais
}

\section{Challenges of the pandemic for mentoring: the role of junior mentors and social networks}

\author{
lana Ciara Santos de Albuquerque 1 (D) ianac@ufrn.edu.br \\ Rebecca Paiva de Araújo Silva' (1) rebeccaufrn@ufrn.edu.br \\ Alice Mendes Duarte ${ }^{1}$ (D) alicemendesduarte@gmail.com \\ Letícia Sousa Oliveira' (D) leticiasousa777@gmail.com \\ Paula Barros de Lins e Silva' (1) paulabarros.ufrn@gmail.com \\ Fabiano César de Medeiros Júnior ${ }^{1}$ (D) fabiano.cesar.jr@gmail.com \\ Nicolas Sartori Viegas ${ }^{1}$ (D) nicolassviegas@gmail.com \\ Simone da Nóbrega Tomaz Moreira' (D) simonetomaz@hotmail.com
}

\section{RESUMO}

Introdução: A aprovação no curso de Medicina traz consigo a euforia e, por vezes, associa-se a grandes mudanças no dia a dia dos alunos. Essas mudanças estão relacionadas a momentos de medo, sofrimento e adaptação. Os programas de mentoria surgem como espaços importantes de cuidado que oferecem acolhimento e suporte às vivências dos alunos, de modo a contribuir para o desenvolvimento pessoal e acadêmico deles.

Relato de experiência: Diante da necessidade de adaptar o ensino presencial ao modelo remoto, em detrimento da pandemia de Covid-19, o programa de mentoria da Universidade Federal do Rio Grande do Norte investiu na realização de atividades on-line, protagonizadas por monitores denominados mentores juniores. Essas atividades se valeram do potencial das mídias sociais, por meio das plataformas de videoconferência, além de interações nas redes sociais, para realizar os encontros e promover a manutenção do vínculo, bem como proporcionar um espaço de acolhimento e integração entre estudantes e mentores.

Discussão: A partir da realização de atividades remotas, alunos e mentores mostraram-se muito participativos e satisfeitos. A atuação dos mentores juniores na elaboração dessas atividades virtuais foi um importante diferencial, possibilitando o engajamento dos mais tímidos e daqueles que tinham dificuldade em cumprir os horários presenciais.

Conclusão: Mediante a observação no desenvolvimento das atividades do programa e com base na avaliação dos alunos e mentores sobre o desempenho da mentoria no semestre remoto, considera-se que é de grande valia investir no potencial das mídias sociais para impulsionar as reuniões dos grupos de mentoria, garantir a manutenção do acolhimento e suporte aos alunos, bem como para estreitar os vínculos entre os participantes. Sob esse prisma, é necessário considerar a possibilidade de adotar um modelo misto no aperfeiçoamento do programa de mentoria.

Palavras-chave: Educação Médica; Mentoria; Estudante Universitário; Isolamento Social; Tecnologia da Informação.

\section{ABSTRACT}

Introduction: Being accepted for medical school brings euphoria, and is sometimes associated to major changes in the student's daily life. These changes pertain to moments of fear, suffering and adaptation. The mentoring programs, therefore, appear as essential care spaces, offering welcoming and support to students, in order to contribute to their personal and academic development.

Experience Report: Faced with the need to adapt from face-to-face teaching to the remote model, to help control the Covid-19 pandemic, the Mentoring program of the Federal University of Rio Grande do Norte invested in conducting online activities led by junior mentors. These activities took advantage of the potential of social media, through video conferencing platforms, in addition to interactions on social networks, to hold the meetings and maintain the link, as well as providing a space for welcoming and integrating students and mentors.

Discussion: In relation to the remote activities, students and mentors were found to be very participative and satisfied. The role of junior mentors in developing these virtual activities was an important differential, enabling the engagement of the most timid and those who had difficulty in meeting the face-to-face schedules.

Conclusion: Through observation during the development of the program activities, and based on the evaluation of students and mentors on the performance of mentoring in the remote semester, the conclusion is drawn that it is important to invest in the potential of social media to boost mentoring group meetings, ensure that the students are offered reception and support, and to strengthen the bonds with the participants. In light of this, it is necessary to consider the possibility of adopting a mixed teaching model to improve the mentoring program.

Keywords: Medical Education; Mentoring; University Student; Social Isolation; Information Technology.

1 Universidade Federal do Rio Grande do Norte, Natal, Rio Grande do Norte, Brasil.

Editora: Patrícia Lacerda Bellodi.

Recebido em 24/02/21; Aceito em 05/04/21.

Avaliado pelo processo de double blind review. 


\section{INTRODUÇÃO}

Os estudantes, ao ingressarem no curso de Medicina, estão geralmente eufóricos e felizes pela conquista da vaga em um concorrido curso universitário. No entanto, o contexto acadêmico exige adaptação à nova realidade, às diferentes metodologias de ensino e aos professores e colegas, o que pode resultar em desafios e angústias ${ }^{1}$. Para alguns estudantes, entrar na universidade significa sair de casa, morar em outra cidade ou em outro estado, e, como consequência, precisam enfrentar a separação familiar. Além disso, a própria natureza do curso de Medicina aproxima os estudantes de situações de dor, sofrimento e morte, e, na maioria dos casos, não reflete sobre os sentimentos advindos dessa realidade ${ }^{2}$.

Os programas de mentoria emergem como espaços de cuidado e interação que oferecem apoio aos estudantes e promovem a reflexão e o acolhimento dos sentimentos e das vivências deles ${ }^{3}$. Esses programas se configuram como uma modalidade de suporte, na qual uma pessoa experiente e empática, o mentor, apoia um jovem iniciante, o mentorado, em seu desenvolvimento pessoal e profissional, de modo a minimizar angústias e promover um ambiente mais propício ao desenvolvimento integral desses alunos ${ }^{4}$.

Na Universidade Federal do Rio Grande do Norte (UFRN), o programa de mentoria teve início no ano de 2015, sendo ofertado como atividade voluntária. No ano seguinte, passou a funcionar como disciplina optativa de 30 horas, com o objetivo de computar formalmente a carga horária para discentes e docentes. Cada turma passou a ser formada por um mentor (professor médico) e por uma média de dez a 15 mentorados (alunos de diversos períodos - distribuídos aleatoriamente nos grupos pela coordenação do curso, visando integrálos e estimular a troca de vivências durante os diferentes períodos) ${ }^{5}$. Nos grupos de mentoria, cada mentor (que também é cadastrado como orientador acadêmico dos discentes do grupo) é responsável por avaliar semestralmente os estudantes, de acordo com a participação nas atividades desenvolvidas.

A partir de 2018, esse programa passou a contar com mentores juniores, que atuam na qualidade de monitores para colaborar na organização das atividades com a coordenação do programa, bem como auxiliar os mentores no planejamento das discussões realizadas nos grupos. A seleção dos mentores juniores se deu por meio de processo seletivo, validado por edital de monitoria da Pró-Reitoria de Graduação, no qual o programa foi contemplado com três bolsas remuneradas e 22 vagas não remuneradas, das quais uma permaneceu vacante. Para que pudessem participar da seleção, os discentes deveriam preencher os seguintes pré-requisitos: ser aluno do curso de Medicina regularmente matriculado na disciplina optativa da mentoria; ter cursado pelo menos dois semestres da mentoria; envio de um plano de trabalho com propostas de atuação como mentor júnior (utilizado para compor a primeira nota da seleção e de caráter eliminatório); e entrevista (utilizada para compor a segunda nota da seleção e de caráter eliminatório). A partir da média, convocaram-se os alunos para que pudessem assumir a monitoria, e os três mais bem classificados foram contemplados com bolsa. Desde então, o programa foi renovado anualmente.

Com a emergência da pandemia da coronavirus disease 2019 (Covid-19) em março de 2020, as atividades do programa de mentoria precisaram ser adaptadas ao novo contexto. Nesse processo de (re)organização, os 24 mentores juniores tiveram um papel decisivo na organização dos 317 discentesmentorados que estiveram distribuídos em 25 grupos: mediar a relação entre mentores e mentorados, e buscar uma maior aproximação com todos os estudantes do curso, na tentativa de minimizar sentimentos de desamparo, angústia, incertezas e temor, oriundos desse período de isolamento social, e até mesmo as dores pelas inúmeras perdas vivenciadas ${ }^{3}$.

Diante das inúmeras mudanças vinculadas ao cenário pandêmico, a educação também teve que se adaptar. A forma de ensinar vem sofrendo mudanças, o que exige de professores e alunos uma maior flexibilidade no processo de ensino-aprendizagem, além da necessidade de adquirir novas habilidades no manejo das ferramentas de tecnologia da informação. Com os grupos de mentoria não foi diferente, alunos e professores adaptaram-se ao formato remoto por meio de aplicativos de videoconferências, redes sociais e outras ferramentas on-line. Inclusive, novas atividades foram propostas visando à construção de um ambiente acadêmico acolhedor e afetivo, apesar do distanciamento social. Assim, este trabalho se propõe a descrever o funcionamento dos grupos, as atividades desenvolvidas e as temáticas discutidas no período da pandemia da Covid-19 pelo programa de mentoria do curso de Medicina da UFRN, destacando-se o "desafio mentoring" e os encontros virtuais da mentoria.

\section{RELATO DE EXPERIÊNCIA}

Em março de 2020, com a suspensão das atividades da UFRN, a comunidade acadêmica ficou com grandes expectativas com relação ao retorno das atividades presenciais. Com o passar do tempo e a impossibilidade do retorno se configurando, a coordenação do programa de mentoria se reuniu com os mentores juniores, por meio do aplicativo Google Meet, a fim de planejar atividades que pudessem ser realizadas, independentemente do retorno das aulas.

Os grupos de mentoria buscaram desenvolver um espaço de aproximação afetiva, de modo a fortalecer o vínculo entre mentores, mentorados e demais envolvidos no curso que não estavam ligados ao programa. Dessa forma, foi estabelecido que 
os mentores juniores iriam propor uma maior interação com os alunos via redes sociais, principalmente pelo Instagram e WhatsApp. Os mentores juniores foram divididos em grupos, e, a partir dessa divisão, cada grupo era responsável pelo desenvolvimento de um desafio para ser proposto aos alunos. Os desafios eram lançados semanalmente, via Instagram e WhatsApp, sendo necessária, por parte do aluno que desejasse participar, a postagem de uma foto no stories do seu perfil do Instagram com a marcação "\#desafiomentoring" e marcando "@tutoriamentoringufrn".

Os desafios semanais desenvolvidos pelos mentores juniores tinham como objetivo estimular os alunos a realizar atividades que não envolvessem necessariamente um estudo do campo médico, encorajando-os a utilizar o tempo livre na quarentena para atividades de lazer e descontração. A utilização da rede social Instagram se deu pela grande popularização dessa mídia entre os matriculados na disciplina. Além disso, uma versão expandida da postagem era inserida no site do programa de mentoria - https://monitoriamentoring.wixsite.com/tutoriaufrn/ divulgacao -, incluindo relatos e dicas dos mentores juniores para motivar os alunos a compartilhar suas experiências.

O Instagram possui mecanismos que possibilitam o cálculo de métricas estimadas, baseadas em uma amostragem ou modelagem estatística, não por contagem direta. Cada postagem apresenta os seguintes elementos a serem analisados: alcance, comentários, compartilhamentos, curtidas e impressões. O marcador de alcance corresponde ao número de usuários da rede social que visualizaram a referida publicação pelo menos uma vez. As impressões são resultado do número de vezes que uma publicação apareceu na tela dos usuários, contabilizando também os casos em que ela aparece mais de uma vez para a mesma pessoa. Os demais elementos, como curtidas, compartilhamentos e comentários, são apenas quantitativos, contabilizando apenas o número de ações que representam na publicação ${ }^{6}$.

O primeiro desafio, postado no dia 22 de maio de 2020, com o tema "Quem é você além da medicina" obteve maior alcance entre as publicações, e, a partir dele, os alunos mostraram diferentes atividades que estavam desenvolvendo no período de isolamento. A segunda publicação foi feita no dia 28 de maio de 2020 com o questionamento "Como você tem se divertido nessa quarentena?", e os alunos compartilharam atividades de leitura, pintura, culinária, decoração, entre outras. A terceira postagem - com o tema "Em movimento na quarentena" aconteceu no dia 9 de junho de 2020 e estimulava os alunos a mostrar suas diferentes formas de praticar atividade física dentro de casa. O quarto desafio - "Como está sua rotina na quarentena?" - ocorreu no dia 18 de junho de 2020 e solicitava aos alunos que mostrassem suas atividades diárias. Nesse desafio, os discentes compartilharam atividades de lazer e pessoais, além de momentos de estudo e organização. O quinto desafio - "Como se manter informado na quarentena?" - ocorreu no dia 26 de junho de 2020 e mostrou as diferentes formas utilizadas pelos acadêmicos para que pudessem se manter informados a respeito de notícias relacionadas à pandemia de Covid-19. O sexto e último desafio - "Quarentena?! Que tal um bom livro?" - foi lançado no dia 2 de julho de 2020. A partir dessa postagem, inúmeros alunos compartilharam os variados estilos de leitura que desenvolveram ou retomaram durante o período de quarentena. Na Tabela 1, pode-se identificar o engajamento das publicações, segundo as análises realizadas pelo próprio Instagram.

Além da estratégia relacionada ao desafio, pôde-se realizar uma live, também no Instagram, sobre saúde mental no contexto da pandemia - assunto abordado de forma recorrente nos desafios -, com o intuito de oferecer suporte emocional aos estudantes, apresentando algumas estratégias de enfrentamento do estresse com a contribuição de profissionais da área da psicologia.

Após três meses de paralisação, a universidade retornou às atividades acadêmicas, por meio de um semestre suplementar com duração de sete semanas, no formato remoto,

Tabela 1. Marcadores disponibilizados pela rede social Instagram para avaliação das publicações relacionadas aos desafios semanais.

\begin{tabular}{|c|c|c|c|c|c|}
\hline Temáticas & Alcance & Comentários & Compartilhamentos & Curtidas & Impressões \\
\hline Quem é você além da medicina? & 484 & 1 & 5 & 52 & 612 \\
\hline Como você tem se divertido nessa quarentena? & 368 & 0 & 6 & 19 & 449 \\
\hline Em movimento na quarentena & 338 & 2 & 1 & 25 & 416 \\
\hline Como está sua rotina na quarentena? & 327 & 0 & 2 & 18 & 389 \\
\hline Como se manter informado na quarentena? & 275 & 0 & 1 & 16 & 320 \\
\hline Quarentena?! Que tal um bom livro? & 238 & 1 & 0 & 17 & 279 \\
\hline
\end{tabular}

Fonte: Elaborada pelos autores com base nos dados extraídos do Instagram. 
sendo voluntário para docentes e discentes. Essa estratégia foi adotada pela universidade com o objetivo de possibilitar a continuidade das atividades letivas, e na tentativa de concluir o primeiro semestre de 2020, para as disciplinas que pudessem ser executadas no formato remoto. Nesse período, o programa aderiu à proposta realizando encontros semanais dos grupos de mentoria no formato virtual. Realizaram-se seis encontros com duração média de uma hora e meia, desenvolvidos por meio das plataformas de videoconferência Google Meet, Zoom e Web Conference. Nesses encontros, cada grupo de mentoria selecionou temáticas de interesse de seus alunos para abordar, a partir de uma metodologia própria e das ferramentas de videoconferência de sua preferência.

Ao fim do período letivo, foi aplicado um questionário on-line com a finalidade de avaliar a satisfação dos alunos com as atividades realizadas, além de identificar a perspectiva e a opinião deles sobre o ensino remoto e as interações por meio do Instagram. Coletaram-se 299 respostas dos alunos, do primeiro ao $11^{\circ}$ período do curso, matriculados no componente curricular da mentoria. A partir do questionamento "Você considera que as atividades virtuais favoreceram a interação no seu grupo?", obteve-se uma resposta positiva em 212 respostas, 42 afirmaram que o modelo virtual não favoreceu as interações do grupo tutorial e 45 respostas consideraram como "indiferente" o modelo adotado.

Ainda de acordo com o formulário, a temática mais discutida entre os grupos de mentoria foi "Saúde mental", seguida de "Hobbies resgatados no período de isolamento social". Abordaram-se temáticas relacionadas à carreira médica, dentro e fora da academia, assim como questões referentes à prática de exercícios físicos, às vivências da pandemia e às perspectivas pós-Covid-19. É importante ressaltar que os alunos poderiam selecionar mais de uma temática, haja vista que foram realizados seis encontros (Tabela 2).

Tabela 2. Frequência de temáticas discutidas nos grupos de mentoria durante o ensino remoto.

\begin{tabular}{lcc}
\hline \multicolumn{1}{c}{ Temáticas } & $\mathbf{n}$ & $\%$ \\
\hline Hobbies & 236 & 78,9 \\
Saúde mental & 234 & 78,2 \\
Produtividade & 202 & 67,5 \\
Perspectivas pós-pandemia & 184 & 61,5 \\
Métodos de estudos & 137 & 45,8 \\
Ética médica & 103 & 34,4 \\
Relação médico-paciente & 99 & 33,1 \\
Exercícios físicos no isolamento social & 76 & 25,4 \\
\hline
\end{tabular}

Fonte: Elaborada pelos autores.
Além desses, outros temas foram desenvolvidos nos grupos de mentoria, tais como: educação financeira, provas de residência, carreira militar, trabalho voluntário, assédio na residência, racismo e carreira médica. A partir da avaliação dos alunos diante da participação do programa por meio das interações e desafios no Instagram, 235 discentes responderam que gostaram das publicações, 13 não gostaram e 51 afirmaram que não utilizam essa rede social.

\section{DISCUSSÃO}

A partir dos desafios que surgiram com a pandemia da Covid-19 e o isolamento social, foi necessário buscar novas estratégias para enfrentamento dessas barreiras. Ademais, a atual crise em curso reforça a necessidade de transformação da educação médica a partir da incorporação de recursos tecnológicos ao processo de ensino $0^{7,8}$. Dessa forma, a utilização de redes sociais e ferramentas de aprendizagem virtual constituíram-se como importantes estratégias para promover a interação entre discentes e docentes, possibilitando a manutenção das atividades da mentoria, seguindo-se, portanto, a tendência de informatização dessas metodologias ${ }^{9}$.

No contexto desse programa de mentoria, além da adaptação dos encontros para o formato virtual, foi necessário utilizar estratégias para buscar a interação e estimular os alunos a compartilhar suas experiências, para que pudessem trabalhar suas próprias limitações, receios e inseguranças. A partir da proatividade dos mentores juniores, foi possível a realização do "desafio mentoring", que possibilitou maior aproximação afetiva entre estudantes, minimizando, inclusive, sintomas psicológicos, muito prevalentes entre estudantes de Medicina² Os desafios semanais desenvolvidos pelos mentores juniores obtiveram grande adesão entre os alunos, trabalhando de maneira lúdica o enfrentamento das limitações advindas do período pandêmico.

Sob a perspectiva de incentivo à saúde mental dos estudantes, alguns autores ressaltam a necessidade de as escolas médicas implementarem estratégias que incentivem o autocuidado e a capacidade de enfrentamento de situações de estresse psicológico ${ }^{10,11}$. Nesse sentido, destacam-se as discussões sobre saúde mental, em que os acadêmicos puderam compartilhar, em seus grupos de mentoria, suas angústias e apreensões; além das diferentes formas de enfrentamento individual trabalhadas, possibilitando o amadurecimento da habilidade da escuta cuidadosa e empática. A partir dessas experiências, alguns alunos teceram comentários:

A abordagem da saúde mental, bastante necessária nesse período crítico, teve um impacto significativo. Só tenho a agradecer! 
O programa de mentoria foi muito importante nesse período conturbado, pois possibilitou ver diversas situações sob a ótica de pessoas diversas, com quem não necessariamente me relacionava, facilitando racionalizar alguns sentimentos e angústias que são inerentes a esse período.

Além dos momentos de partilha, foram desenvolvidas também atividades lúdicas, com a prática de mindfulness, jogos virtuais e rodas de conversa, para discussão literária e cinematográfica. Tais intervenções visaram reduzir a ansiedade e o estresse, intensificados durante esse período, promovendo melhoria na sensação subjetiva de bem-estar ${ }^{12}$.

É importante destacar que, durante o período remoto, os mentores exerceram papel fundamental ao oferecerem apoio e atenção aos estudantes, especialmente diante das angústias relativas aos impactos da pandemia na graduação. Também é válido enfatizar a maior participação e interação entre alunos diante do meio virtual, o que denotou satisfação diante da utilização das plataformas digitais para a manutenção das atividades da mentoria. Nesse sentido, destacaram-se alguns depoimentos feitos pelos participantes do programa:

Esse modelo virtual de mentoria é bastante cômodo [...] na minha opinião, poderia ser adotado um modelo misto de encontros virtuais e presenciais mesmo após a pandemia.

A forma virtual dos encontros possibilitou uma maior participação dos integrantes da mentoria, o que rendeu maior interatividade e boas conversas.

Esses depoimentos espelham o que alguns autores já vêm trabalhando durante a pandemia, ao mostrarem que diferentes projetos de mentoria podem ser convertidos em um formato on-line, com adesão e resultados semelhantes aos eventos presenciais ${ }^{11,13}$. Ademais, é importante ressaltar o protagonismo que os mentores juniores obtiveram ao proporem e executar essas novas estratégias, incorporando ao programa uma vertente que atrela à educação médica novas tecnologias e processos de ensino. Nesse processo, foram adquiridas competências transversais, a exemplo do trabalho em equipe e contribuições em temáticas socioculturais, além do incentivo à descoberta de novas habilidades que os discentes poderiam desenvolver durante a pandemia ${ }^{14}$.

Quanto à atuação dos mentores juniores no suporte aos demais mentorados, seja nos seus respectivos grupos ou no "desafio mentoring", é sabido que a modalidade de "mentoria de pares", na qual essa relação de suporte é estabelecida diretamente entre alunos, também é bastante difundida em escolas médicas. Essa relação de suporte, quando instituída entre discentes, propicia naturalmente o estabelecimento de um diálogo mais horizontal, diferentemente do que ocorre quando o aluno se dirige diretamente ao mentor docente, pois, por existir ainda uma arraigada ideologia com relação à hierarquia de títulos, pode haver certo constrangimento ${ }^{15,16}$.

Em nosso contexto, apesar de o curso de Medicina da UFRN adotar a modalidade de mentoria em grupo, evidencia-se o protagonismo do mentor júnior como um elo entre a figura do mentorado e a do mentor médico, para estreitar as relações entre discentes e docentes, permitindo compreensão mútua, fortalecimento do vínculo entre colegas e estabelecimento de espaços acolhedores e seguros.

\section{CONCLUSÃO}

O programa de mentoria da UFRN tem seis anos de atividade e, ao longo desse tempo, contribuiu para a formação pessoal e profissional dos estudantes de Medicina dessa instituição. Apesar da conjuntura da pandemia de Covid-19, o programa perseverou e passou por adaptações para conseguir cumprir seus objetivos.

A necessidade do distanciamento social trouxe consigo desafios para a mentoria. Para transpô-los, por meio das redes sociais, desenvolveram-se formas inovadoras de interagir com seus alunos, destacando-se o "desafio mentoring" e a adaptação dos encontros para o formato virtual, os quais foram fatores fundamentais para o estreitamento de laços

No tocante aos "desafios mentoring", eles possibilitaram momentos de autorreflexão e de construção de novos hábitos nos diferentes âmbitos da saúde: físico, mental e social. Com relação aos encontros, a individualidade de cada grupo de mentoria foi expressa pelos diversos temas escolhidos a partir dos interesses dos alunos. Com a pandemia, os problemas de saúde mental se exacerbaram, e a mentoria foi fundamental para realizar o acolhimento dos discentes.

É válido ressaltar ainda a relevância dos mentores juniores na idealização, no planejamento e na execução das atividades propostas na disciplina, bem como a atuação deles para o estabelecimento de uma satisfatória relação mentormentorado. $\mathrm{O}$ novo modelo exigiu um maior conhecimento sobre as plataformas digitais disponíveis, possibilitando a escolha entre elas e a sua utilização de acordo com a adequação à atividade proposta.

Por conseguinte, nota-se que o novo padrão, dotado de adaptações condizentes com o momento atual, permitiu a continuação do programa de modo satisfatório, auxiliando no crescimento integral dos estudantes. Foi perceptível também o grande potencial das ferramentas digitais em possibilitar a realização de atividades do programa de mentoria, com considerável aceitabilidade e elogios pelos alunos envolvidos. Além disso, é certo que a articulação e proatividade dos mentores juniores foram essenciais para o desenvolvimento 
do projeto e para o sucesso obtido, o que reitera a importância da atuação do aluno na construção de uma educação médica individualizada e cada vez mais efetiva.

\section{CONTRIBUIÇÃO DOS AUTORES}

Todos os autores trabalharam na sistematização das atividades do programa de mentoria durante o período de ensino remoto, que serviram de base para elaboração deste trabalho, bem como participaram da escrita do manuscrito, do delineamento do relato de caso, do levantamento bibliográfico e da problematização da discussão.

\section{CONFLITO DE INTERESSES}

Declaramos não haver conflito de interesses.

\section{FINANCIAMENTO}

Declaramos não haver financiamento.

\section{REFERÊNCIAS}

1. Moreira SNT, Vasconcellos RLSS, Heath N. Estresse na formação médica: como lidar com essa realidade? Rev Bras Educ Med. 2015;39(4):558-64.

2. da Costa DS, Medeiros NSB, Cordeiro RA, Frutuoso ES, Lopes JM, Moreira SNT. Sintomas de depressão, ansiedade e estresse em estudantes de Medicina e estratégias institucionais de enfrentamento. Rev Bras Educ Med. 2020;44(1):1-10.

3. Moreira SNT, de Albuquerque ICS, Pinto Junior FEL, Gomes AHB. Programa de mentoria do curso de Medicina da Universidade Federal do Rio Grande do Norte: atividades integrativas em foco. Rev Bras Educ Med. 2020;44(4):1-6.

4. Bellodi PL, Martinho T, Massaroppe B, Martins MA, Santos MAS. Temas para um programa de tutoria em Medicina: uma investigação das necessidades dos alunos da FMUSP. Rev Bras Educ Med. 2004; 28(2):119-27.
5. Chaves LJ, Gonçalves ECQ, Ladeira LR, Ribeiro MS, Costa MB, Ramos AAM. A tutoria como estratégia educacional no ensino médico. Rev Bras Educ Med. 2014;38(4):532-41.

6. Instagram. Sobre métricas estimadas, em desenvolvimento e de terceiros [acesso em 21 fev 2021]. Disponível em: https://www.facebook.com/help/ instagram/2937306082959298.

7. Tabatabai S. Covid-19 impact and virtual medical education. J Adv Med Educ Prof. 2020 July;8(3):140-3.

8. McKimm J, Ramani S, Kusurkar RA, Fornari A, Nadarajah VD, Thampy H, et al. Capturing the wisdom of the crowd: health professions' educators meet at a virtual world café. Perspect Med Educ. 2020 Dec 1;9(6):385-90.

9. de Albuquerque ICS, Coelho JFR, Antunes JPL, do Nascimento CD, de Lima DLT, Costa RDS, et al. Construção de um dicionário virtual para incremento do aprendizado de Biologia no ensino propedêutico. Revista Brasileira de Informática na Educação. 2015;23(03):131-43.

10. Kazerooni AR, Amini M, Tabari P, Moosavi M. Peer mentoring for medical students during the Covid-19 pandemic via a social media platform. Med Educ. 2020;54(8):762-3.

11. Guse J, Heinen I, Kurre J, Mohr S, Bergelt C. Perception of the study situation and mental burden during the Covid-19 pandemic among undergraduate medical students with and without mentoring. GMS J Med Educ. 2020;37(7):1-9.

12. Luken M, Sammons A. Systematic review of mindfulness practice for reducing job burnout. Am J Occup Ther. 2016;70(2):7002250020p1$7002250020 p 1013$.

13. Gernert JA, Zibold J, Reik LJU, Graupe T, Dimitriadis K. Restructuring career counselling ventures of a mentoring program for medical students in the course of the Covid-19 pandemic. GMS J Med Educ. 2020;37(7):1-6.

14. Gonçalves MCN, Bellodi PL. Ser mentor em medicina: uma visão arquetípica das motivações e transformações na jornada. Interface Comun Saúde Educ. 2012;16(41):501-14.

15. de Souza MG, Reato LFN, Bellodi PL. Ressignificando a relação entre calouros e veteranos: mentoria de pares na visão de alunos mentores. Rev Bras Educ Med. 2020;44(4):1-9.

16. Akinla $\mathrm{O}$, Hagan $\mathrm{P}$, Atiomo W. A systematic review of the literature describing the outcomes of near-peer mentoring programs for first year medical students. BMC Med Educ. 2018;18(1):167-77. 